\title{
Das Projekt SwissDRG nimmt Form an und legt an Tempo zu: Die FMH ist ein wichtiges Rad in diesem Prozess
}

Kürzlich hat der Bundesrat mehrere Änderungen in den Ausführungsverordnungen zum KVG (KVV, VKL) erlassen. Dies hat zur Folge, dass nur eine einzige Stelle berechtigt sein wird, die Tarifstruktur der Spitäler im Sozialversicherungsbereich zu verwalten und zu verbessern: Dies legitimiert und stärkt die Rolle der SwissDRG AG. Ausserdem

\section{Nur eine einzige Stelle ist berechtigt, die Tarif- struktur der Spitäler im Sozialversicherungsbereich zu verwalten und zu verbessern: Dies legitimiert und stärkt die Rolle der SwissDRG AG}

wurde der Handlungsbereich dieser zu Beginn des Jahres von den Partnern des Gesundheitswesens - unter anderem von der FMH - gegründeten Gesellschaft auf die Geriatrie, die Rehabilitation und die Psychiatrie ausgedehnt. Zugleich geben diese Verordnungen zwingende Fristen vor, was das neu geschaffene CaseMix Office (CMO) von Anfang an unter Zeitdruck setzt und vom Verwaltungsrat Entscheidungen verlangt.

Der wichtigste Termin ist der 30. Juni 2009: An diesem Datum muss die neue Tarifstruktur dem Bundesrat zur Genehmigung eingereicht werden. Da es aufwendig ist, nachträglich noch grössere Anpassungen vorzunehmen, drängt sich die Ausarbeitung eines möglichst weit entwickelten ersten Produkts auf. Die FMH, die sich dessen bewusst ist, hat die Entwicklung vorhergesehen und die Voraussetzungen geschaffen, um den Helvetisierungsprozess 2008 konsequent zu begleiten. Die Vertreter unserer Fachgesellschaften und Dachverbände, die für diese Herausforderung sensibilisiert sind, haben die Botschaft sehr gut aufgenommen - so gut, dass die FMH dem CMO kürzlich über
1600 Verbesserungsanträge einreichen konnte. Sie alle sollten in die Prozendurenklassifikation $\mathrm{CHOP}$ und in die Version 0.2 des SwissDRG-Groupers einfliessen, die das Gerüst der ersten Tarifstruktur bildet, die der Landesregierung eingereicht wird. Zudem hatten die Autoren die Gelegenheit, ihre Anträge den von uns beauftragten Experten zu präsentieren: Mit ihren fundierten Ratschlägen konnten die Experten die spezifischen Kenntnisse der Spezialisten durch eine Gesamtsicht der DRG-Mechanik ergänzen. Dieses sehr sorgfältige Vorgehen erlaubte es, die Begründung für jeden Fall noch breiter abzustützen. Dies verleiht der Ärzteschaft hohe Glaubwürdigkeit - und sie wird nun vom CMO für die anschliessende Analyse dieser Anträge direkt einbezogen.

Gerne nutze ich die Gelegenheit, den Fachgesellschaften und Dachverbänden für die enorme Arbeit zu danken, die sie diesen Herbst geleistet haben. Damit haben Sie der FMH ermöglicht, jenen Kritikern, die immer wieder versuchen, unseren Einfluss zu beschränken, die bestmögliche

\section{Die FMH konnte dem CMO kürzlich über 1600 Verbesserungsanträge einreichen. Dieses Vorgehen verleiht der Ärzteschaft hohe Glaubwürdigkeit}

Antwort zu geben: Die Ärzteschaft ist kompetent, kooperativ, konstruktiv, speditiv und vor allem unentbehrlich für die Erarbeitung einer glaubwürdigen Tarifstruktur im Spitalbereich.

Dr. med. Pierre-François Cuénoud, Mitglied des FMH-Zentralvorstands, Leiter Ressort SwissDRG 